\title{
A type of periodicity of certain quartic surfaces
}

\author{
By W. L: EDGE. \\ (Received 3rd September, 1941. Read 1st November, 1941.)
}

\section{Introduction}

The following pages have been written in consequence of reading some paragraphs by Reye ${ }^{1}$, in which he obtains, from a quartic surface, a chain of contravariant quartic envelopes and of covariant quartic loci. This chain is, in general, unending; but Reye at once foresaw the possibility of the quartic surface being such that the chain would be periodic. The only example which he gave of periodicity being realised was that in which the quartic surface was a repeated quadric. It is reasonable to suppose that, had he been able to do so, he would have chosen some surface which had the periodic property without being degenerate; in the present note two such surfaces are signalised.

In $\S 1$ we define, for a form of any even degree in any number of variables, the contravariant which is the generalisation of Reye's contravariant of a quartic surface, and we allude to the question of periodicity in $\S 2$. The periodicity of a certain quartic surface is then established in $\S 3$. The periodicity of a second quartic surface is established by a different method in $\S 6$; this method makes use of a new definition of Reye's contravariant, and this definition is obtained in $\S 5$.

1. The polar forms of order $p$ of a form $F$ of order $2 p$ in $n$ variables are linear combinations of its different $p$ th derivatives. When these derivatives, $\frac{(n+p-1) !}{(n-1) ! p !}$ in number, are written beneath one another in successive rows, the coefficients of the homogeneous products of degree $p$ in the variables $x_{i}$ form a square matrix $\mu$. The number of polar forms of order $p$ which are linearly independent is equal to the rank of $\mu$, and it is supposed that $\mu$ has its full rank, so that its determinant, which is an invariant of $F$, does not vanish.

\footnotetext{
1 Journal für Math. 82 (1877), 1!8.
} 
It is convenient to clear up two points, and thereby ensure that $\mu$ is symmetric.

In the first place, while the sequence in which the derivatives of $F$ follow one another may be chosen arbitrarily, it is supposed that the homogeneous products of degree $p$ follow one another in the sequence which corresponds exactly to that chosen for the derivatives.

This matching of the two sequences, one of the derivatives.and one of the homogeneous products, does not of itself secure the symmetry of $\mu$; it has to be supplemented by a rule concerning the coefficients. We suppose $F$ to be written out with the appropriate multinomial multipliers preceding its literal coefficionts. Then, if the $p$ th derivatives are each multiplied by $p ! /(2 p)$ !, they also will have appropriate multinomial multipliers, appropriate, that is, to forms of order $p$. To form the matrix $\mu$ we take constant multiples (1) of these derivatives and select from them the coefficients of certain constant multiples (2) of the homogeneous products, observing the following rule: if

$$
C_{i_{1} i_{2} \ldots \ldots i_{n}} \frac{p !}{(2 p) !} \frac{\partial^{p} F}{\partial x_{1}^{i_{1}} \partial x_{2}^{i_{2}} \ldots \partial x_{n^{n}}^{i^{2}}}
$$

and

$$
C_{i_{1} i_{2} \ldots i_{n}}^{\prime} x_{1}^{i_{1}} x_{22}^{i} \ldots x_{n}^{i_{n}}
$$

are a polar form and its associated homogeneous product, then, for all values of the $i^{\prime}$ 's such that $i_{1}+i_{2}+\ldots+i_{n}=p$,

$$
C_{i_{1} i_{2} \ldots \ldots i_{n}} C_{i_{1} i_{2} \ldots \ldots i_{n}}^{\prime}=\frac{p !}{i_{1} ! i_{2} ! \ldots \ldots i_{n} !} .
$$

Then $\mu$ is a symmetric matrix.

This being so, let $\mu$ be bordered by a row and column consisting of the homogeneous products, of degree $p$, of variables $u_{i}$ contragredient to the $x_{i}$; the sequence of products is again to correspond exactly to that of the derivatives, and the coefficients which multiply these products of contragredient variables are to be precisely those numbers $C$ which appear in (1). Then the determinant of this bordered matrix is a contravariant ${ }^{1} R$, of class $2 p$, of $F$.

While the coefficients in (1) and (2) may be arbitrary so long as they satisfy (3), it is convenient, and adds to the symmetry, to fix

1 The proof of the invariance of $|\mu|$ and of the contravariance of $R$, which has been given in Proc. Royal Soc. Edinburgh, A 61 (1941), 157, for the case of $p=2$, can be carried over, with the few necessary verbal changes, to furnish a proof for any value of $p$. 
them so that corresponding coefficients $C$ and $C^{\prime}$ are always equal. This will be done in what follows, so that now

$$
i_{1} ! i_{2} ! \ldots i_{n} ! C_{i_{1} i_{2} \ldots \ldots i_{n}}^{2}=p !
$$

2. When $p=1$ the derivation of the contravariant $R$ is familiar; $F=0$ is the equation of a quadric in point coordinates while $R=0$ is the equation of the same quadric in prime coordinates. If the procedure dual to that by which $R$ was derived from $F$ is now applied to $R$, the resulting determinant has the value $|\mu| F$, and we return thereby to the equation of the quadric in point coordinates. But when $p>1$ this phenomenon of periodicity no longer presents itself unless $F$ is particularised.

We suppose henceforward that $p=2$, so that $F=0$ is the equation of a quartic locus and $R=0$ the equation of a contravariant quartic envelope. The dual procedure, applied to $R$, yields a second quartic locus $F_{1}=0$, a covariant of, and in general distinct from, $F=0$. What is the characteristic property which $F$ must possess in order that the two quartic loci, $F=0$ and $F_{1}=0$, should be the same?

For $n=3$ this question becomes an enquiry concerning plane quartic curves, and was considered by Coble ${ }^{1}$. But, for $n=4$, it was surely in the mind of Reye when, in 1876, he wrote the paper occupying pp. 173-206 of Vol. 82 of the Journal für Math. Reye it was who first found the contravariant $R$ for a quartic surface, and he immediately foresaw the possibility that the chain of quartic loci and envelopes so derived from $F$ might be periodic. Moreover he actually demonstrated that $F_{1}=0$ is the same surface as $F=0$ when this quartic surface is a repeated quadric ${ }^{2}$. In these pages, without attempting to give a complete answer to the question, it is shown that this happens also for two non-degenerate quartic surfaces:

(i) the surface $x^{4}+y^{4}+z^{4}+t^{4}+12 x y z t=0$,

(ii) the surface generated by the tangents of a-twisted cubic.

1 Trans. American Math. Soc. 4 (1903), 65-85.

2 The proof which Reye gives (p. 200) of this result in space of three dimensions is. easily extended to give the correspondingr result in space of any number of dimensions. 
3. We obtain the Reye contravariant, in contragredient variables $u, v, w, p$ of the quaternary form

$$
\left[F \equiv x^{4}+y^{4}+z^{4}+t^{4}+12 x y z t\right.
$$

by direct evaluation of the determinant $R$.

Let the sequence of homogeneous products of degree 2 be

$$
x^{2}, y^{2}, z^{2}, t^{2}, y z, z x, x y, x t, y t, z t ;
$$

the sequence of second derivatives of $F$ must correspond with this. Any arbitrariness in the choice of coefficients is removed by (4). If we write $\tau^{2}=2$ the seventh row, for example, of $\mu$ consists of the coefficients of

$$
x^{2}, y^{2}, z^{2}, t^{2}, \tau y z, \tau z x, \tau x y, \tau x t, \tau y t, \tau z t
$$

in the quadratic form

$$
\tau \cdot \frac{2 !}{4 !} \cdot \frac{\partial^{2} F}{\partial x \partial y} \equiv \tau z t
$$

Thus

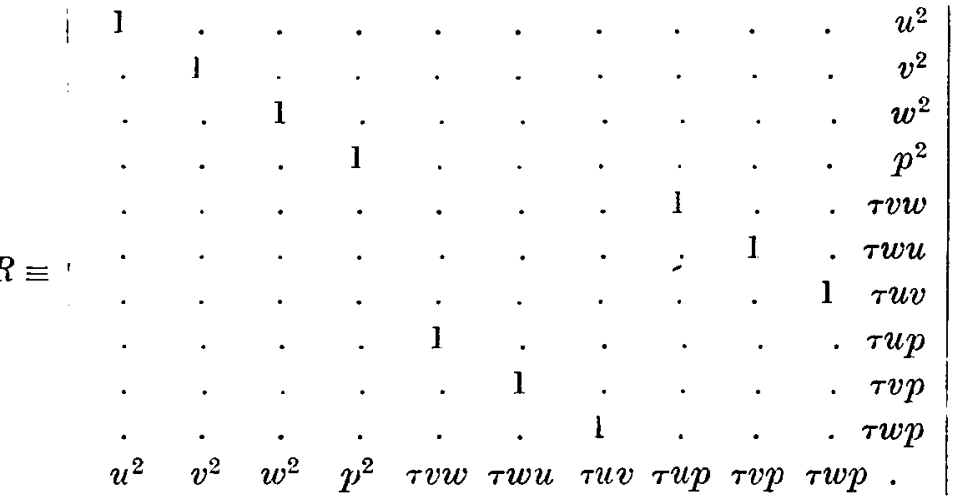

If we expand, by Laplace's rule, on rows $5,6,7,8,9,10$ we obtain, in addition to

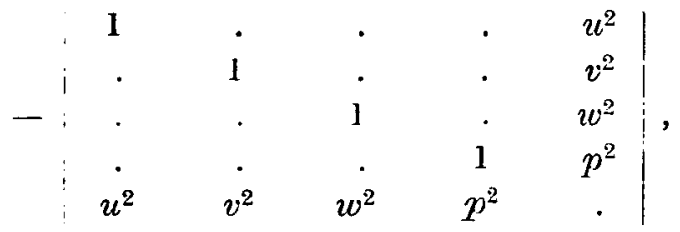

only six non-vanishing terms, each of which is equal to $\tau^{2} u v w p$. Hence

$$
R \equiv u^{4}+v^{4}+w^{4}+p^{4}+12 u v w p
$$


Thus $R$ is precisely the same function of $u, v, w, p$ as $F$ is of $x, y, z, t$; and the covariant $F_{1}$, derived from $R$ by the process dual to that by which $R$ was derived from $F$, is identical with $F$ itself.

It may also be easily verified that, if

$$
F \equiv \alpha x^{4}+\beta y^{4}+\gamma z^{4}+\delta t^{4}+12 \lambda x y z t,
$$

then $R \equiv a \beta \gamma \delta \lambda^{6}\left(\alpha^{-1} u^{4}+\beta^{-1} v^{4}+\gamma^{-1} w^{4}+\delta^{-1} p^{4}+12 \lambda^{-1} u v w p\right)$,

and $\quad F_{1} \equiv\left(\alpha \beta \gamma \delta \lambda^{6}\right)^{8}\left(\alpha x^{4}+\beta y^{4}+\gamma z^{4}+\delta t^{4}+12 \lambda x y z t\right)$.

Thus here again $F_{1}=0$ is the same surface as $F=0$.

Perhaps a word may be interpolated as to why the particular quartic surface $F \equiv x^{4}+y^{4}+z^{4}+t^{4}+12 x y z t=0$ was selected. The reason was that this surface is invariant for a large group of collineations'. Its Reye contravariant envelope, and the covariant surface $F_{1}=0$, must therefore be invariant for the same group; and so it seemed, to say the least, extremely likely that the quartic surfaces $F=0$ and $F_{1}=0$ might be the same.

III

4. The polar quadric of any point $(X, Y, Z, T)$ with respect to the quartic surface $F(x, y, z, t)=0$ is

$$
X^{2} \frac{\partial^{2} F}{\partial x^{2}}+Y^{2} \frac{\partial^{2} F}{\partial y^{2}}+\ldots \ldots \ldots+2 Z T \frac{\partial^{2} F}{\partial z \partial t}=0 .
$$

If $(X, Y, Z, T)$ is constrained to lie on a quadric $Q$ a linear relation is thereby imposed on the squares and products of its four coordinates; hence, as the point varies on $Q$, the quadrics (A) vary within a linear system of freedom 8 , and so are all outpolar to a unique quadric envelope. This holds for any quadric $Q$. In particular $Q$ may be a repeated plane $\pi$, in which case let the inpolar envelope be called $\Sigma$; $\pi$ does not, in general, belong to $\Sigma$; the envelope of those planes $\pi$ which do belong to their associated envelopes $\Sigma$ is a contravariant of $F$. This is Reye's contravariant; and it can be seen from Reye's work how to obtain the determinantal form of its equation from this geometrical definition.

This definition of the contravariant, emerging, as it does, from a $(1,1)$ correspondence between quadric loci and quadric envelopes set up through the agency of the quartic surface, is an attractive one; yet for some purposes it is profitable to use another which starts

I Burnside: The Theory of Groups (Second Edition, Cambridge, 1911), 371, Ex. 6. 
immediately from the plane $\pi$ and which involves only linear systems of quadrics.

5. If two points are given, $F$ may be polarised in succession by means of them; the order in which the two polarisations are carried out is immaterial, since the same quadric is obtained. This quadric is the mixed polar of the pair of points; in particular, were the two points to coincide at $P$, the quadric would be the second polar of $P$ with respect to $F=0$.

The mixed polar of any two points $P_{1}, P_{2}$ that are conjugate with respect to a quadric $Q$ belongs to the pencil determined by the second polars of the two points in which $P_{1} P_{2}$ meets $Q$, and this pencil is equally well determined by the mixed polars of any two pairs of conjugate points on $P_{1} P_{2}$. There are $\infty^{5}$ pairs of points conjugate with respect to $Q$; their mixed polars determine the same linear system of quadrics as do the second polars of all the points of $Q$.

Now let $Q$ be a repeated plane $\pi$. The $\infty^{5}$ pairs of points that are conjugate for this degenerate quadric are got by pairing each of the $\infty^{2}$ points of $\pi$ with each of the $\infty^{3}$ points of space. The mixed polar, however, of a pair of points being the polar quadric of either with respect to the cubic surface which is the first polar of the other, the $\infty^{5}$ mixed polars are those quadrics which are the first polars of all the points of space with respect to those cubic surfaces which are themselves the first polars, with respect to $F=0$, of the points of $\pi$. If then there exists a quadric envelope $\Sigma$ inpolar to all these $\infty^{5}$ mixed polars, it must be inpolar to every first polar of each of the cubic surfaces and so, to use the common idiom, inpolar to the cubic surfaces themselves.

The existence and uniqueness of $\Sigma$ follow, of course, from the corresponding known results when $Q$ is non-degenerate; but they can be established independently by elementary considerations. For let $P_{1}, P_{2}, P_{3}$ be any three non-collinear points of $\pi ; C_{1}, C_{2}, C_{3}$ the cubic surfaces which are their respective first polars. The first polars of all the points of space with respect to the cubic surfaces constitute three linear systems $\sigma_{1}, \sigma_{2}, \sigma_{3}$, of quadrics, each of freedom 3 . But any two of $\sigma_{1}, \sigma_{2}, \sigma_{3}$ have one quadric in common; the quadric common to $\sigma_{2}$ and $\sigma_{3}$, for example, is the mixed polar of $P_{2}$ and $P_{3}$, which is both the first polar of $P_{2}$ with respect to $C_{3}$ and the first polar of $P_{3}$ with respect to $C_{2}$. It follows that $\sigma_{1}, \sigma_{2}, \sigma_{3}$ are contained in a linear system of quadrics of freedom 8 , and therefore that all their members are outpolar to a unique quadric envelope $\Sigma$. Hence the alternative 
definition of $R$, established in its own right and independently of the preceding one:

The first polars of the points of a plane $\pi$ with respect to $F=0$ constitute a net of cubic surfaces, and there is a unique quadric envelope $\Sigma$ which is inpolar to them all. In general, $\pi$ does not belong to $\Sigma$; the envelope of those planes $\pi$ which do belong to their associated envelopes $\Sigma$ is Reye's contravariant.

This definition, and the descriptive argument by which it has been established, can be at once extended to space of any number of dimensions.

\section{IV}

6. Suppose now that $F$ is the surface generated by the tangents of a twisted cubic $\Gamma$. The net of first polars of the points of a plane $\pi$ is linearly dependent on the first polars of the three intersections $P_{1}, P_{2}, P_{3}$ of $\pi$ with $\Gamma$. Let $P_{1}$ and $P_{2}$ be the first and last vertices of the tetrahedron of reference for a system of homogeneous coordinates $(x, y, z, t)$; this system can be set up so that $\Gamma$ is given parametrically by $\left(\theta^{3}, \theta^{2} \phi \sqrt{ } 3, \theta \phi^{2} \sqrt{ } 3, \phi^{3}\right)$. It is not strictly necessary to introduce the $\sqrt{ } 3$ here; but it has been done because its introduction gives a consequent parametric form for the coordinates of an osculating plane of $\Gamma$ which is precisely the same as that for the coordinates of a point of $\Gamma$.

The equation of $F$ is

$$
(y z-3 x t)^{2}=4\left(y^{2}-z x \sqrt{ } 3\right)\left(z^{2}-y t \sqrt{ } 3\right),
$$

and the first polars of $P_{1}$ and $P_{2}$ are

$$
2 z^{3}=3 \sqrt{ } 3\left(y z t-x t^{2}\right) \text { and } 2 y^{3}=3 \sqrt{ } 3\left(x y z-x^{2} t\right) .
$$

It is then found that those quadric envelopes which are inpolar to both these cubic surfaces are obtained by varying the constants $\alpha, \beta, \gamma$ in the equation

$$
a\left(v^{2} \sqrt{ } 3+4 w u\right)+\beta(2 v w+u p)+\gamma\left(w^{2} \sqrt{ } 3+4 v p\right)=0 ;
$$

and that the quadric $\Sigma$ which belongs to this net and which is inpolar also to the first polar of the third intersection of $\pi$, whose equation may be taken as $\phi y=\theta z$, with $\Gamma$ is

$$
\theta^{2}\left(v^{2} \sqrt{ } 3+4 w u\right)+2 \theta \phi(2 v w+u p)+\phi^{2}\left(w^{2} \sqrt{ } 3+4 v p\right)=0 .
$$


The condition for $\pi$ to belong to $\Sigma$ is

$$
\theta^{2} \phi^{2}=0 \text {. }
$$

It follows that, if $P_{1}, P_{2}, P_{3}$ are distinct points, $\pi$ cannot belong to $\Sigma$. Therefore, first, the contravariant $R$ does not vanish identically and, secondly, it must be the envelope of those planes which touch $\Gamma$. The surface enveloped by these planes has an equation, in plane coordinates, obtained from the equation of $F$ simply by writing $u, v, w, p$ instead of $x, y, z, t$ respectively. And so, when the process dual to that by which the envelope was obtained from $F$ is applied to the envelope, the result is to return to the quartic surface $F$.

Mathematical Institute,

The University,

EDINBURGH. 